\title{
A conductive mechanism of PVA (Mowiol 10-98) filled with ZnO and MWCNT nanoparticles
}

\author{
N B RITHIN KUMAR ${ }^{1, *}$, VINCENT CRASTA ${ }^{2}$, B M PRAVEEN ${ }^{3}$ and B GANANATH SHETTY ${ }^{4}$ \\ ${ }^{1}$ Department of Physics, A J Institute of Engineering and Technology, Mangalore 575006, India \\ ${ }^{2}$ Department of Physics, St Joseph Engineering College, Vamanjoor, Mangalore 575028, India \\ ${ }^{3}$ Department of Chemistry, Srinivas School of Engineering, Mukka, Mangalore 574146, India \\ ${ }^{4}$ Department of Physics, Alva's College, Moodbidri 574227, India \\ *Author for correspondence (rithin4u@ rediffmail.com)
}

MS received 21 February 2018; accepted 15 November 2018; published online 25 April 2019

\begin{abstract}
Polyvinyl alcohol (PVA) hybrid nanocomposites are prepared via an ex situ approach with ZnO and MWCNT nanoparticle fillers and their conductive mechanisms have been investigated. The tailored hybrid nanocomposite conformation and their microstructural disparities for different filler concentrations were studied using an X-ray diffractometer. The direct current (DC) conductivity studies show an increase in the conductivity from $1.0528 \times 10^{-11}$ to $2.1514 \times 10^{-8} \mathrm{~S} \mathrm{~cm}^{-1}$ up to a percolation threshold filler concentration of $x=7.5 \mathrm{wt} \%$. The dielectric constant substantially indicates a decreasing trend with increasing frequency. The exaggerated dielectric constant values of $11.8 \mathrm{at} 5 \mathrm{kHz}, 6.3 \mathrm{at} 100 \mathrm{kHz}, 5.86 \mathrm{at}$ $500 \mathrm{kHz}$ and 2 at $1 \mathrm{MHz}$ are observed for $7.5 \mathrm{wt} \%$ filler hybrid nanocomposites, which indicates their potential application as a gate material in metal-oxide-semiconductor field-effect transistors (MOSFETs). The alternating current (AC) electrical conductivity demonstrates an increasing behaviour up to $x=7.5 \mathrm{wt} \%$ filler concentration. The smaller values observed in the real part of the electric modulus $\left(M^{\prime}\right)$ indicates a riddance in electrode polarization. The observed higher frequency shift in the imaginary part of the electric modulus for increasing the filler concentration up to $x=7.5 \mathrm{wt} \%$, decreases the relaxation time of the dipole orientation thereby increasing the conductivity mechanism of the hybrid nanocomposites. Apart from these, its small relaxation time with high electrical conductivity favours this material PVA/ $(x) \mathrm{MWCNT}(15-x) \mathrm{ZnO}$ to have prospective application in microwave absorption appliances. The increase in the surface roughness of the film seen from the AFM images up to $x=7.5 \mathrm{wt} \%$ concentration supports an enhancement in the crystalline nature of the filler. Differential scanning calorimeter studies show an enhancement in glass transition temperature $\left(T_{\mathrm{g}}\right)$, melting temperature $\left(T_{\mathrm{m}}\right)$ and decomposition temperature $\left(T_{\mathrm{d}}\right)$ for PVA filled with MWCNTs and $\mathrm{ZnO}$ composites for optimum filler concentration $x=7.5 \mathrm{wt} \%$.
\end{abstract}

Keywords. Nanocomposite; filler; PVA.

\section{Introduction}

Nowadays, the addition of metal oxide nanoparticles into a polymer matrix has become a unique metal-oxygen combination to enhance the physical and structural properties of polymer nanocomposites. These metal-oxygen bonds as the main backbone consisting of side chains, such as organic groups, hydrides or halogens revolutionize the nanocomposites for the fabrication of optical, dielectric, electrical and opto-electronic devices [1-4]. In this contemporary era, zinc oxide $(\mathrm{ZnO})$ nanoparticles, which is the combination of zinc and oxygen metal-oxide atoms, have proved to be the best filler in terms of optical and electrical properties for polymer hybrid nanocomposites. These $\mathrm{ZnO}$ metal oxide nanoparticles have less energy gap and some outstanding properties, such as antibacterial and anticorrosive with high chemical stability [5-7]. On the other hand, multi-walled carbon nanotubes (MWCNTs) were also proved to be ideal filler candidates inside a polymer matrix. The potential applications are due to their high aspect ratio with length having tremendous mechanical strength and conducting behaviour. These gifted characteristics of MWCNTs in the polymer matrix aid in the formulation of electrical, opto-electronic and dielectric commercial devices [8-10].

In this current work, we report on the preparation of PVA hybrid nanocomposites using polyvinyl alcohol (PVA, Mowiol 10-98) as the host polymer matrix. PVA has an ability to form a broad range of hydrogen bonds and this helps the PVA to bind with the added fillers for the preparation of novel hybrid nanocomposites [11-13]. Furthermore, the basic host PVA matrix is filled with MWCNTs and $\mathrm{ZnO}$ nanoparticles. This mixed filler combination ( $\mathrm{ZnO}$ and MWCNTs) inside a single PVA matrix leads to the formation of unique organic-inorganic hybrid nanocomposites having the effects of both the fillers in the solitary polymer matrix. Thus, by mixing two fillers in a single polymer matrix, we can anticipate improvements in their physical characteristics promoting new applications in materials science. The PVA hybrid nanocomposites are traditionally prepared using the 
solvent casting method at room temperature. Furthermore, these $\mathrm{PVA} /(x) \mathrm{MWCNT}(15-x) \mathrm{ZnO}$ nanocomposites for $x=0,1,5,7.5,10,14$ and $15 \mathrm{wt} \%$ concentration hybrid nanocomposites are characterized to study their structural, dielectric relaxation, relaxation time for dipole orientation, electric conductivity and thermal conductivity behaviour.

\section{Experimental}

\subsection{Materials}

The basic host PVA (Mowiol 10-98) fully hydrolysed to 97-100 mol\% grade was provided by Sigma Aldrich, Germany. The procured PVA has a molecular weight, $M_{\mathrm{w}}=$ $61,000 \mathrm{~g} \mathrm{~mol}^{-1}$, degree of polymerization, $P_{\mathrm{W}}=1400$ and bulk density ranging from 0.4 to $0.6 \mathrm{~g} \mathrm{~cm}^{-3}$. The reagents for the production of $\mathrm{ZnO}$ nanoparticles were provided by Merck, India. The additional filler MWCNTs $(75 \mathrm{mg})$ were obtained from Sigma Aldrich, Germany (CVD, 95\% purity). The chemicals used for the preparation of PVA hybrid nanocomposites were procured from Merck, India.

\subsection{Preparation of PVA hybrid nanocomposites}

Initially, $\mathrm{ZnO}$ nanoparticles are synthesized by the precipitation method to act as a filler [14]. The additional filler MWCNTs (carbon nanotube, multi-walled $>98 \%$ carbon 6-8 tube walls) are functionalized with the carboxyl group by treating with sulphuric acid $\left(\mathrm{H}_{2} \mathrm{SO}_{4}\right)$ and nitric acid $\left(\mathrm{HNO}_{3}\right)$ taken in a 3:1 volume ratio solution and sonicated by using a probe ultrasonicator for $2 \mathrm{~h}$. Using the nanofillers of $\mathrm{ZnO}$ and MWCNTs, the PVA/ $(x) \operatorname{MWCNT}(15-x) \mathrm{ZnO}$ hybrid nanocomposite solvents are prepared by sonication of the composite solvent for $x=0,1,5,7.5,10,14$ and $15 \mathrm{wt} \%$ filler concentrations. The each filled composite solvent mixture was stirred and sonicated until the hybrid composite solvent was bubble free, homogeneous and highly viscous. The obtained solvent composite for various filler concentrations is casted on a Petri dish glass. This solvent is allowed to evaporate at room temperature for 3 days and peeled off. The average thickness of the prepared PVA hybrid nanocomposites was measured using a dial thickness gauge (Mitutoyo, Japan) and was found to be 100-130 $\mathrm{m}$.

\subsection{Measurements}

X-ray diffraction (XRD) data of PVA/ $(x) \operatorname{MWCNT}(15-x)$ $\mathrm{ZnO}$ nanocomposites were chronicled using a Bruker D8 Advance $\mathrm{X}$-ray diffractometer with Ni filtered, $\mathrm{CuK} \alpha$ radiation of wavelength $\lambda=1.5406 \AA$, with a graphite monochromator and a scanning speed of $2^{\circ} \mathrm{min}^{-1}$ in the $2 \theta$ range of $10-110^{\circ}$. From the $I-V$ characteristics, the direct current (DC) conductivity studies of the prepared PVA nanocomposites were analysed using a Keithley 4200-SCS parameter analyser through the computer interface by the two-probe method at room temperature. The $I$ vs. $V$ (current vs. voltage) measurements were performed in the voltage range of $0-5 \mathrm{~V}$ in multiple steps of $0.1 \mathrm{~V}$. The frequency-dependent parameters such as dielectric properties and capacitance were analysed using a Keithley 4200-SCS parameter analyser at room temperature in the frequency range of $1 \mathrm{kHz}-1 \mathrm{MHz}$. The composite surface topography related to roughness and particle size was investigated using a Bruker (Nano) atomic force microscope (AFM). Thermal studies were performed using a differential scanning calorimeter (DSC) Q2000 from TA Instruments.

\section{Results and discussion}

\subsection{XRD studies}

XRD spectra of the PVA loaded with different concentrations of MWCNTs and $\mathrm{ZnO}$ show intense peaks at $2 \theta=32.33$, 35.15, 36.93, 48.49, 57.66, 64.23, 67.67, 69.35, 70.46 and $74.29^{\circ}$ signposts for filled $\mathrm{ZnO}$ nanoparticles (ICSD collection code: 154490 and International Center for Diffraction Data, JCPDS 5-0664) and a small peak of the loaded MWCNT at $2 \theta=26.52^{\circ}$ (JCPDS file no: 48-1449). The broad prominent peaks of PVA are observed between $2 \theta=19$ and $20^{\circ}$ [15]. The enhancement in the filler concentration leads to deviations in shifts and intensity of the crystalline peaks of PVA spectra around $2 \theta=19^{\circ}<2 \theta<$ $22^{\circ}$. The observed shifts and variations in the intensity suggest the changes in the actual degree of order or crystalline phases of the PVA nanocomposites. Figure 1 shows that the increase in the concentration of fillers up to $x=7.5 \mathrm{wt} \%$ intensifies the crystalline peaks of PVA and the peaks shift towards the lower angle. The crystallizability of the PVA nanocomposites is found for $x=7.5 \mathrm{wt} \%$ filler. These facts suggest that the PVA chains during solidification take up the conformation leading to highly ordered and oriented chain aggregates with the added nanoparticles. This strong interfacial interaction occurring between the added filler up to $x=7.5 \mathrm{wt} \%$ with the host PVA transforms the polymer nanocomposites to gain a high impact on physical properties. For $x>7.5 \mathrm{wt} \%$, the crystalline peaks of PVA shift nearer to the higher angle with a decrease in the intensity resulting in a low crystalline phase in the PVA matrix. This happens because the increase in carbon atoms and the decrease in oxygen vacancies of the fillers reduce the hydrogen bonding and ionic bonding and coordination interactions with the host PVA matrix. As a result, the nanoparticles cause less agglomeration in PVA nanocomposites thereby decreasing the physical properties $[16,17]$.

\subsection{DC conductivity}

The following relationship provides the measurements of DC conductivity of the PVA nanocomposites tailored with MWCNTs and ZnO nanofillers,

$$
\sigma_{\mathrm{DC}}=\frac{d}{R A},
$$




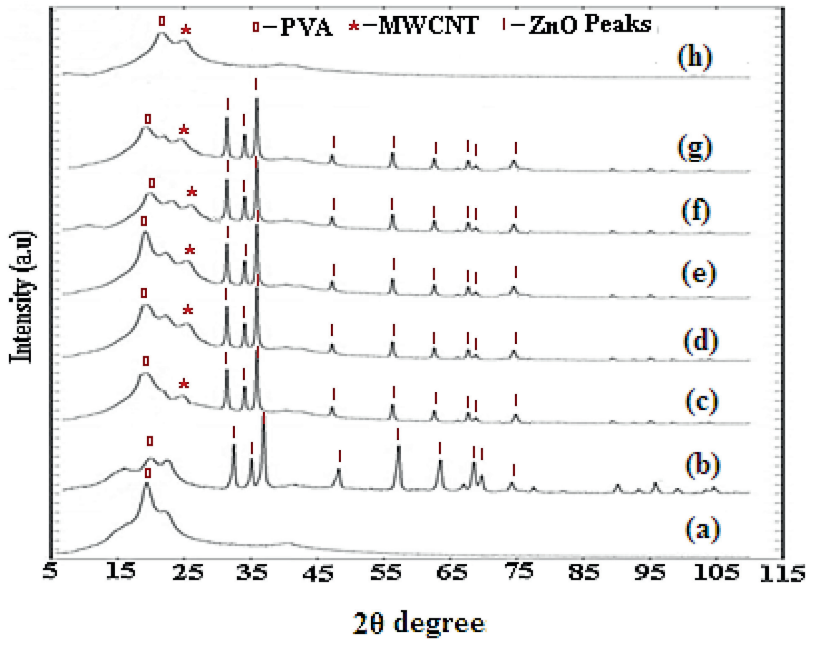

Figure 1. XRD peaks of PVA/ $(x) \mathrm{MWCNT}(15-x) \mathrm{ZnO}$ nanocomposites: (a) pure PVA, (b) $x=0 \mathrm{wt} \%$, (c) $x=1 \mathrm{wt} \%$, (d) $x=$ $5 \mathrm{wt} \%$, (e) $x=7.5 \mathrm{wt} \%$, (f) $x=10 \mathrm{wt} \%$, (g) $x=14 \mathrm{wt} \%$ and (h) $x=15 \mathrm{wt} \%$.

Table 1. Variations of DC conductivity for different filler concentrations of PVA/( $x) \mathrm{MWCNT}(15-x) \mathrm{ZnO}$.

\begin{tabular}{lccc}
\hline S. no. & $\begin{array}{c}\text { Sample } \\
(\text { wt } \%)\end{array}$ & Resistance $(\Omega)$ & $\begin{array}{c}\text { Conductivity } \\
(\sigma \text { in S cm }\end{array}$ \\
\hline 1 & Pure PVA & $9.4984 \times 10^{6}$ & $1.0528 \times 10^{-11}$ \\
2 & 0 & $3.7617 \times 10^{6}$ & $4.6583 \times 10^{-10}$ \\
3 & 1 & $2.3528 \times 10^{4}$ & $4.2502 \times 10^{-9}$ \\
4 & 5 & $1.264 \times 10^{4}$ & $7.9063 \times 10^{-9}$ \\
5 & 7.5 & $0.4648 \times 10^{4}$ & $2.1514 \times 10^{-8}$ \\
6 & 10 & $3.7895 \times 10^{5}$ & $2.6388 \times 10^{-10}$ \\
7 & 14 & $11.895 \times 10^{5}$ & $8.406 \times 10^{-11}$ \\
8 & 15 & $2.7486 \times 10^{4}$ & $3.6382 \times 10^{-9}$ \\
\hline
\end{tabular}

where $d$ is the thickness of the sample, $A$ the cross-sectional area of the electrode used and $R$ the resistance offered by the sample, which is assessed from the $I-V$ data.

Table 1 shows the measurements of DC conductivity of $\mathrm{PVA} /(x) \mathrm{MWCNT}(15-x) \mathrm{ZnO}$ nanocomposites. The DC conductivity is found to increase with the increase in the filler concentration up to $x=7.5 \mathrm{wt} \%$ and above $x=7.5 \mathrm{wt} \%$, the values show a decreasing trend. The increase in the filler concentration up to the threshold level reduces the entanglement length thereby reducing the volume fraction of nanoparticles. The threshold ratio of carbon and oxygen molecules receding in the interstitial position of PVA molecules assists them to form charge transfer complexes through hydrogen bonding between the filled nanoparticles and host PVA molecules. The increase in the filler concentration up to the threshold limit causes the increase in charge transfer complexes. This reduces the barrier height and provokes the hopping of charges from one charge complex to other, significantly increasing the conductivity [18]. Thus, the formation of the percolate network of the conducting filler inside the PVA/ $(x) \mathrm{MWCNT}$ (a)

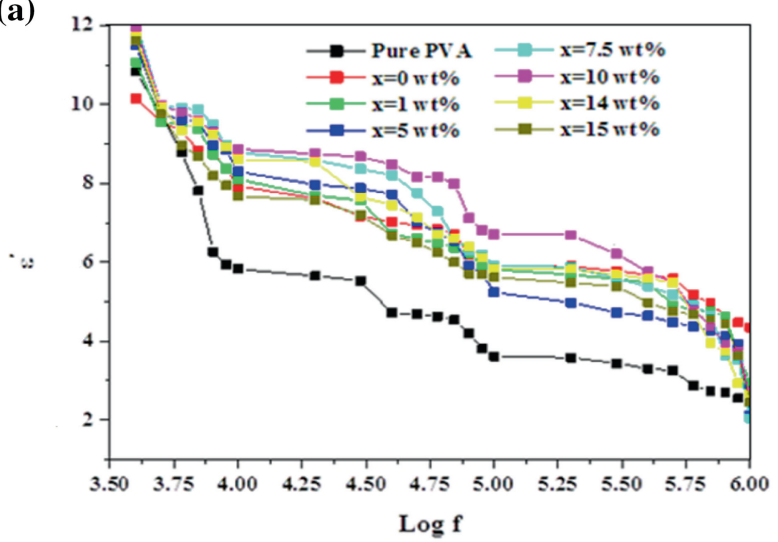

(b)

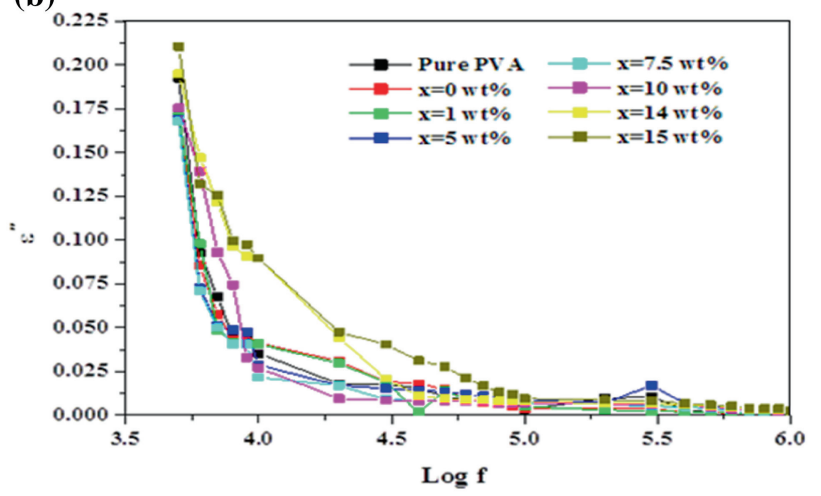

Figure 2. Variations of (a) real and (b) complex dielectric constants with an increase in the frequency of PVA/ $(x) \operatorname{MWCNT}(15-x)$ $\mathrm{ZnO}$ nanocomposites for different ' $x$ ' filler concentrations.

$(15-x) \mathrm{ZnO}$ nanocomposites up to the optimized filler concentration $(x=7.5 \mathrm{wt} \%)$ increases the electrical conductivity.

The incorporation of nanoparticles above the threshold filler $(x>7.5 \mathrm{wt} \%)$ causes the variation in the ratio of carbon and oxygen molecules in the PVA molecule. The small variation in the filler concentration leads to a significant change in the tethered zone and tether chain conformation. This leads to the creation of agglomeration in PVA nanocomposites reducing the charge transfer complexes thereby reducing conductivity.

\subsection{Dielectric studies}

The dielectric studies were carried out in the frequency range of $1 \mathrm{kHz}-1 \mathrm{MHz}$ isothermally at room temperature. Figure $2 \mathrm{a}$ and $b$ represents the significant variation of dielectric constant $\left(\varepsilon^{\prime}\right)$ and dielectric loss $\left(\varepsilon^{\prime \prime}\right)$ with the increase in the frequency range of $1 \mathrm{kHz}-1 \mathrm{MHz}$.

The defect density in the forbidden gap is responsible for the variation in the real and imaginary parts of the dielectric constant of the hybrid nanocomposite films [19-21]. The observed higher values of the real part of the dielectric constant at a lower frequency range signposts the greater 


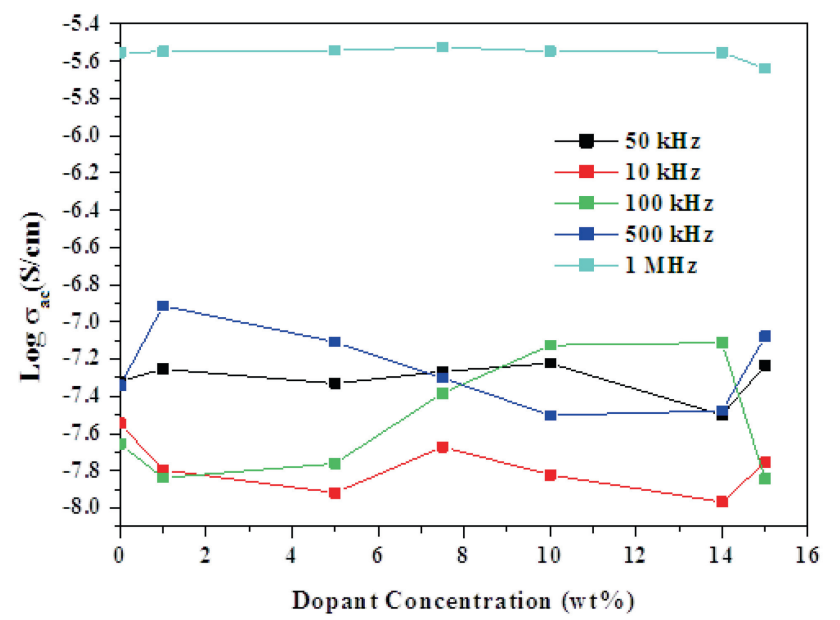

Figure 3. $\mathrm{AC}$ conductivity values of $\mathrm{PVA} /(x) \operatorname{MWCNT}(15-x)$ $\mathrm{ZnO}$ for different filler concentrations.

energy storage capacity of the hybrid PVA nanocomposites. On the other hand, the decrease in the dielectric constant with the increase in the frequency indicates the polar molecular nature of nanocomposites. This strange behaviour is due to the interfacial effect observed in the grain boundaries caused by the immovability of charges which hampers the molecular motion. As a result, the dipoles themselves start to orient in the applied field direction. With the increase in the frequency, the movement of bound charges is promoted and the dipole orientation in the applied field direction is reduced. Thus, the transport of bound charges encourages the enhancement in conductivity with the increase in the frequency $[18,22]$.

\subsection{AC conductivity}

The following relationship is utilized to determine the AC conductivity performance of the synthesized polymer nanocomposites,

$$
\sigma_{\mathrm{AC}}=2 \pi f \varepsilon_{0} \varepsilon^{\prime \prime}
$$

where $f$ is the applied frequency, $\varepsilon_{0}$ the permittivity of free space and $\varepsilon^{\prime \prime}$ the dielectric loss.

Figure 3 shows the AC electrical conducting mechanism of the filled PVA nanocomposites. The frequency dependence of the electrical behaviour of materials is explained by:

$$
\sigma_{\mathrm{AC}}=\sigma_{\mathrm{DC}}+\sigma_{1}(\omega),
$$

or

$$
\sigma_{\mathrm{AC}}=\sigma_{\mathrm{DC}}+A \omega^{n}
$$

where $\sigma_{\mathrm{DC}}$ is the frequency independent DC conductivity, $\sigma_{1}(\omega)$ the typically assigned to the hopping conduction, $A$ the pre-exponential factor dependent on the temperature and $n$ the exponent ( $n<1$, the hopping motion is a translational one whereas $n>1$, the motion is a localized one).
According to the law, the following effects that contribute to the AC conductivity are:

(1) electrode effect (active low frequencies),

(2) DC plateau (at intermediate frequencies),

(3) defect process (which can be explained by the term $\left.A \omega^{n}\right)$ viz., quantum mechanical tunnelling through the barrier separating the localized state or correlated barrier hopping over the same barrier.

The improvement in the AC electrical conductivity was found up to $x=7.5 \mathrm{wt} \%$ filler concentration. It has also been observed that the conductivity increases with frequency and is characteristic of the $\omega^{n}$ ( $n$ is the exponential). Furthermore, the Jonscher power law is applied for all the prepared composites and is confirmed by the non-linear fit. It was observed that at low frequencies, there is a slight deviation for all the composites. The non-linear fit also suggested that the value of $n<1$ corresponds to the diffusion limit. Hence, the transport mechanism is explained by the hopping process between the two sites separated by an energy barrier.

Apart from these, two mix fillers i.e., $\mathrm{ZnO}$ and MWCNT nanoparticles are filled on the host PVA matrix. The conduction may also occur by the formation of MWCNT and $\mathrm{ZnO}$ nanoparticle networks. This can be explained based on the primary particle model. The conductive mechanism shows a discrepancy by means of the electron tunnelling mechanism as explained above. This quantum tunnelling is a governing mechanism around the percolation threshold. The quantum mechanical tunnelling represents the wave function of the electron that is not restricted completely within a potential box, but also contains a small tail prolonging beyond the potential barrier. This small tail helps the stream of conduction electrons $\left(10^{-5}-10^{-10}\right)$ having kinetic energy below the potential barrier to penetrate the barrier and reach the next aggregate $[18,24]$. The increase in the filler concentration gradually reduces the distance between the filled nanoparticles and enhances the carbon and oxygen molecules encouraging the conductance in the nanocomposites. At a threshold ratio of $x=7.5 \mathrm{wt} \%$ filler concentration, the distance between the nanoparticles is $<10 \mathrm{~nm}$ and the conductivity escalates owing to the tunnelling effects. Furthermore, increase in the filler concentration above $x=7.5 \mathrm{wt} \%$ decreases the carbon and oxygen vacancy ratios and also when the content of the filled particles is above the percolation threshold, the conducting mechanism decreases by the overlapping of wave functions and ohmic instead of the tunnelling process. The decrease in the filler ratio reduces the free ion concentration and assists in the less agglomeration process of fillers leading to a larger particle having a reduced surface area. These factors lead to the reduction in the conductivity for the filler concentration above $x=7.5 \mathrm{wt} \%$ [10,25].

Figure 4 shows the intensification in the $\mathrm{AC}$ electrical conductivity with frequency due to dielectric transition. These 


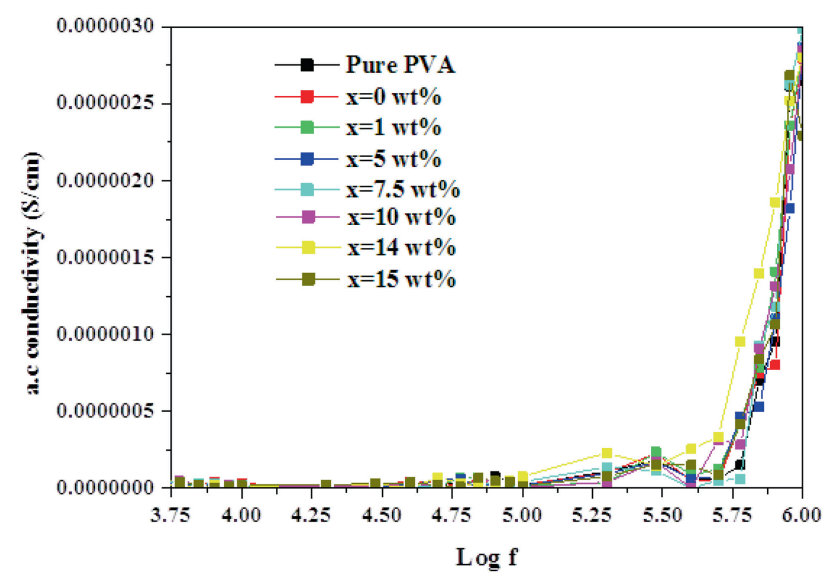

Figure 4. Variations of $\mathrm{AC}$ conductivity with frequency of $\mathrm{PVA} /(x) \mathrm{MWCNT}(15-x) \mathrm{ZnO}$ nanocomposites for different ' $x$ ' nano-filler concentrations.

transitions generate chemical changes in each PVA molecule improving the $\mathrm{AC}$ conducting behaviour of nanocomposites [10,25-29].

\subsection{Electric modulus}

The electric modulus $(M)$ in the frequency domain is determined by the reciprocal of the complex dielectric $[10,25,29]$

$$
M=\frac{1}{\varepsilon^{\prime}+i \varepsilon^{\prime \prime}}=M^{\prime}+i M^{\prime \prime},
$$

where $M^{\prime}=\varepsilon^{\prime} /\left(\varepsilon^{\prime 2}+\varepsilon^{\prime \prime 2}\right)$ epitomizes the real part and $M^{\prime \prime}=$ $\varepsilon^{\prime \prime} /\left(\varepsilon^{\prime 2}+\varepsilon^{\prime \prime 2}\right)$ symbolizes the imaginary part of the electric modulus.

The imaginary part of the electric modulus represents the measure of distribution of ion energies and determines the value of dipole relaxation time $\left(\tau_{\mathrm{P}}\right)$ using the following equation:

$$
\tau_{\mathrm{P}}=\frac{1}{\omega_{\mathrm{P}}},
$$

where $\omega_{\mathrm{p}}$ is the angular frequency obtained from asymmetric peaks of the imaginary part of electric modulus $\left(M^{\prime \prime}\right) v s$. frequency graph.

The observed small values of the real part of the electric modulus at a lower frequency in figure 5a epitomize the high dielectric constant [19].

These small values of the real part of the electric modulus $\left(M^{\prime}\right)$ represent the restricted orientation of dipoles and the elimination of electrode polarization $[10,29]$. Figure 5a shows an S-shaped curve indicating the increased values of $M^{\prime}$ with the increase in frequency. This asymptotic value of $M^{\prime}$ results from short-range mobility of charge carriers during conduction [30].
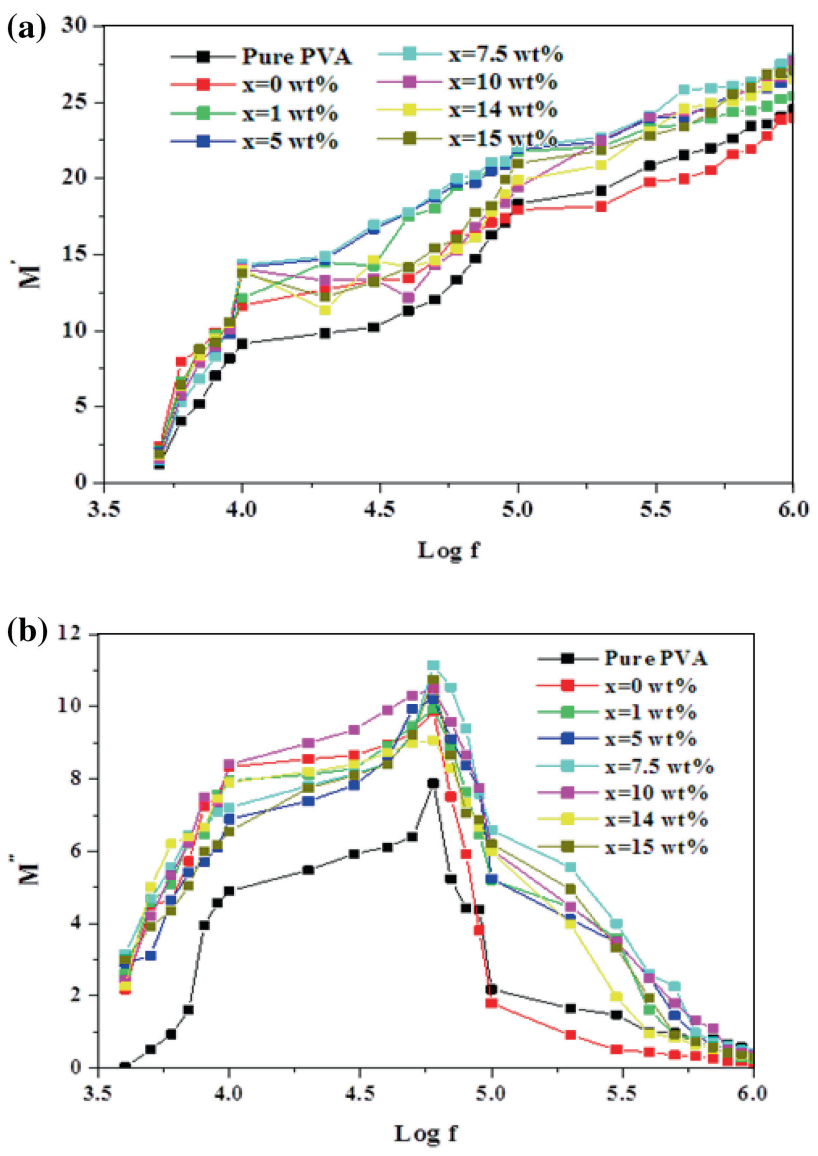

Figure 5. Variations of (a) real and (b) complex electric moduli with an increasing frequency of PVA/ $(x) \operatorname{MWCNT}(15-x) \mathrm{ZnO}$ nanocomposites for various ' $x$ ' nano-filler concentrations.

Figure $5 \mathrm{~b}$ represents the variation of the imaginary part of the electric modulus $\left(M^{\prime \prime}\right)$ with the increase in the applied frequency. The observed distinct asymmetric peaks in $M^{\prime \prime}$ are obtained from the heterogeneous polymeric system having a different dielectric constant. A noticeable shift in the maximum asymmetric peaks with the increase in filler concentration up to $7.5 \mathrm{wt} \%$ reveals Maxwell-Wagner-Sillars (MWS) polarization [31].

This polarization restricts the relaxation time of the dipole orientation and promotes the hopping of charges from one site to other. These favourable facts authorize the local chain dynamics of the PVA hybrid nanocomposites and enhance their conductivity on the filler.

Furthermore, the increase in the filler concentration above $x=7.5 \mathrm{wt} \%$ allows the asymmetric peaks to shift towards a lower value. This fact supports the presence of localized sites consisting of short-range motion of charge carriers with increasing relaxation time. The increase in the relaxation time reduces the transition of charge carrier mobility plummeting the electrical conductivity of the prepared hybrid nanocomposites. 

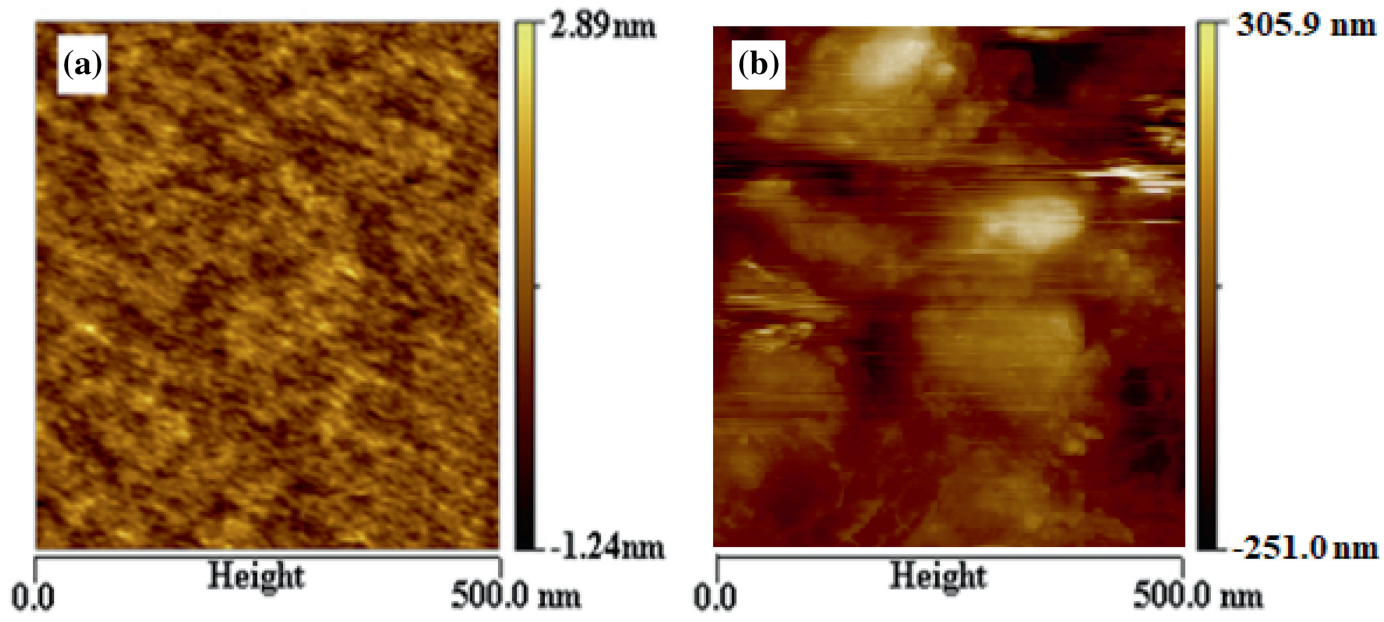

Figure 6. AFM 2-D images of (a) pure PVA and (b) MWCNT- filled PVA for $7.5 \mathrm{wt} \%$ concentration.

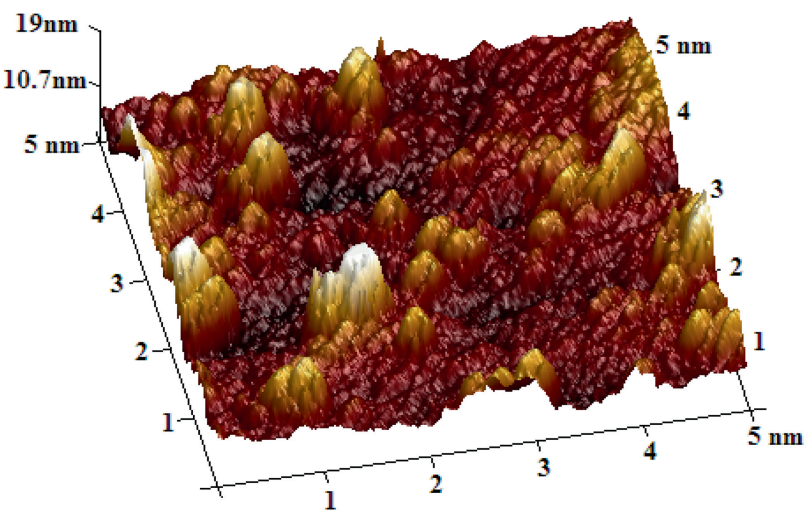

Figure 7. AFM 3-D image of $\mathrm{PVA} /(x) \mathrm{MWCNT}(15-x) \mathrm{ZnO}$ for $x=7.5 \mathrm{wt} \%$.

\subsection{Film topography}

Figure $6 \mathrm{a}$ and $\mathrm{b}$ represents the AFM two-dimensional images of pure and $x=7.5 \mathrm{wt} \%$ filled hybrid nanocomposites. Figure 7 shows the three-dimensional AFM topography of the tailored PVA hybrid nanocomposites for the optimum percolation threshold filler concentration $(x=7.5 \mathrm{wt} \%)$. The AFM photographs of the pure PVA film show a smooth surface having reduced grain size. The addition of fillers into the PVA matrix changes the grain size and obviously roughens the surface of the hybrid nanocomposites due to the presence of undulation between valleys and peaks that are present. The transport properties and fouling potential also depend on the composite surface roughness. The difference among the highest peak $\left(Z_{\max }\right)$ and lowest valley $\left(Z_{\min }\right)$ on the composite surface provides the peak to valley roughness. The average peak to valley roughness $\left(R_{\text {avg }}\right)$ for the obtained AFM image is $54.9 \mathrm{~nm}$. This observation is due to the adsorption of PVA molecules tightly on the surface of nanoparticles. These primary complex particulate formations are due to the result of the particle-particle interactions, as the added nanoparticles are completely encapsulated by PVA, which impressively shields or interdicts the strong interactions between the filled nanoparticles. Thus, the adsorption of polymer main chains with loaded nanoparticles results in complex formation i.e., host PVA molecular chains may adsorb mixed nanoparticles to form the nano-sized hybrids [32]. This wrapping shell of PVA covering the whole nanofillers changes the root mean square (rms) values [33]. Thus, change in the filler concentration attributes the variations to the rms value, which indicates the fluctuations in the crystallinity of the prepared hybrid nanocomposites. On the other hand, the AFM data represented in table 2 regarding the complex particle parameters support the fact that the prepared hybrids are of nano-size in nature.

\subsection{DSC studies}

Figure 8 presents the effects of thermal behaviour of unfilled and filled PVA for optimized filler concentration $x=7.5 \mathrm{wt} \%$ in the temperature range of $30-350^{\circ} \mathrm{C}$. The recognizable increase in the $T_{\mathrm{g}}$ from 49.10 to $56.17^{\circ} \mathrm{C}$ for $x=7.5 \mathrm{wt} \%$ concentration indicates the formation of the complex through the interaction of fillers with the hydroxyl group of the PVA backbone.

The influence of other functional groups also supports the hydrogen bonding and enhances the value of $T_{\mathrm{g}}$ to a maximum for the optimum filler concentration $x=7.5 \%$. The width of the $T_{\mathrm{g}}$ for the filled concentration of the polymer nanocomposites is almost identical to that of the unfilled PVA components signifying the single-phase behaviour of the polymer nanocomposites. The endothermic peak occurring at $220.66^{\circ} \mathrm{C}$ was accredited to the melting point of the PVA nanocomposites [34]. The increase in the value of $T_{\mathrm{m}}$ for $x=7.5 \mathrm{wt} \%$ filler concentration signifies the increase in the crystallite size in the crystalline phase or the increase in the order of the molecular packing in the crystallites. On the other hand, the dispersion of nanoparticle fillers into the PVA main 
Table 2. AFM particle size parameters of PVA/(x)MWCNT $(15-x) \mathrm{ZnO}$ for $x=7.5 \mathrm{wt} \%$ filler concentration.

\begin{tabular}{lrrrr}
\hline Parameter & \multicolumn{1}{c}{ Mean } & Minimum & Maximum & \multicolumn{1}{c}{ Sigma } \\
\hline Total count & 12.000 & 12.000 & 12.000 & 0.000 \\
Density $\left(\mu \mathrm{m}^{-2}\right)$ & 0.030 & 0.030 & 0.030 & 0.000 \\
Height $(\mathrm{nm})$ & 108.003 & 23.181 & 224.744 & 77.661 \\
Area $\left(\mathrm{nm}^{2}\right)$ & 779215.500 & 158691.406 & 3808593.750 & 992543.563 \\
Diameter $(\mathrm{nm})$ & 869.520 & 449.502 & 2202.102 & 485.863 \\
\hline
\end{tabular}

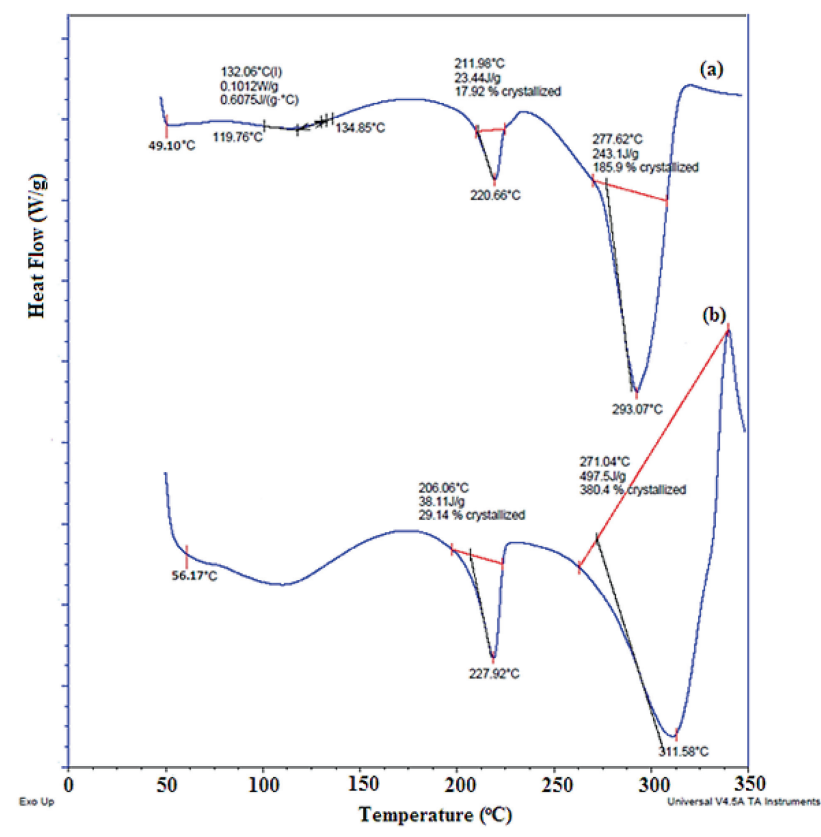

Figure 8. DSC thermograph of (a) pure PVA and (b) PVA with MWCNTs and $\mathrm{ZnO}$ for $x=7.5 \mathrm{wt} \%$ filler concentration.

chain causes a tendency of local ordering in the PVA structure. This occurrence of local ordering might be due to the intramolecular and inter-molecular interactions existing between the nanoparticle fillers and PVA structure. Thus, these factors increase the size of the crystallite in the composite film. Figure 8 also shows that the addition of nanoparticle fillers also enhances the decomposition temperature $\left(T_{\mathrm{d}}\right)$ and further on heating the PVA nanocomposites above the $T_{\mathrm{d}}$, the polymer composite initiates a rapid chain-stripping elimination of $\mathrm{H}_{2} \mathrm{O}$ causing colour changes and crosslinking to yield insoluble yellow to black rigid foam-like residues as it decomposes $[35,36]$.

\section{Conclusions}

The fascinating results of the conductive mechanism of the novel PVA/ $(x)$ MWCNT $(15-x) \mathrm{ZnO}$ hybrid nanocomposites for different percolation filler concentrations are studied. The
XRD studies confirm the hybrid PVA chain entanglements, variation in the crystalline properties and other microstructural disparities with the increase in the filler concentration. It is found that for percolation threshold, filler concentration $x=7.5 \mathrm{wt} \%$, the enhancement in the crystalline properties is achieved. The hybrid nanocomposites featured for up to $x=7.5 \mathrm{wt} \%$ percolation filler concentration show a steep increase in DC conductivity from $1.0528 \times 10^{-11}$ to $2.1514 \times 10^{-8} \mathrm{~S} \mathrm{~cm}^{-1}$. The high dielectric constant values at low frequency indicate the electrode polarization and with the increase in frequency, the dielectric shows a decreasing trend. At a threshold ratio for $x=7.5 \mathrm{wt} \%$ filler concentration, due to the presence of oxygen and carbon molecules and also due to tunnelling effects, the conductivity of the hybrid nanocomposites increases. The exaggerated dielectric constant values of 11.8 observed at $5 \mathrm{kHz}, 6.3$ at $100 \mathrm{kHz}$, 5.86 at $500 \mathrm{kHz}$ and 2 at $1 \mathrm{MHz}$ are noticed for $7.5 \mathrm{wt} \%$ filler hybrid nanocomposites indicating their potential application as a gate material in metal-oxide-semiconductor field-effect transistors (MOSFETs). The smaller values of the real part of the electric modulus $\left(M^{\prime}\right)$ signify the elimination of electrode polarization. The observed higher frequency shift in the imaginary part of the electric modulus $\left(M^{\prime \prime}\right)$ for an increasing filler concentration up to $x=7.5 \mathrm{wt} \%$ decreases the relaxation time of the dipole orientation, thereby increasing the conductivity of the nanocomposites. Apart from these, its small relaxation time with high electrical conductivity favours this material PVA/ $(x) \mathrm{MWCNT}(15-x) \mathrm{ZnO}$ to have prospective application in microwave absorption appliances. The addition of fillers into the PVA matrix changes the grain size and enhances the surface roughness of the hybrid nanocomposites which supports the enhancement in the crystalline nature of the PVA nanocomposites up to $x=7.5 \mathrm{wt} \%$ percolation threshold, which are analysed by AFM. The DSC studies show an enhancement in $T_{\mathrm{g}}$, melting temperature $\left(T_{\mathrm{m}}\right)$ and $T_{\mathrm{d}}$ for PVA filled with MWCNTs and $\mathrm{ZnO}$ composites for optimum filler concentration $x=7.5 \mathrm{wt} \%$.

\section{References}

[1] Balamurugan A, Ho K C and Chen S M 2009 Synth. Met. 159 2544 
[2] de Barros R A, Martins C R and de Azevedo W M 2005 Synth. Met. 15535

[3] Rajeswari N, Selvasekarapandian S, Karthikeyan S, Sanjeeviraja C, Iwai Y and Kawamura J 2013 Ionics 191105

[4] Wang Z L, Kong X Y, Ding Y, Gao P, Hughes W L, Yang R et al 2004 Adv. Funct. Mater. 14943

[5] Ando M, Kadono K, Haruta M, Sakaguchi T and Miya M 1995 Nature 374625

[6] Kumar N B R, Crasta V and Praveen B M 2016 Mater. Res. Express. 3055012

[7] Mardare D and Rusu G I 2004 J. Optoelectron. Adv. Mater. 6 333

[8] Jariwala D, Sangwan V K, Lauhon L J, Marks T J and Hersam M C 2013 Chem. Soc. Rev. 422824

[9] Zhou Y X, Freitag M, Hone J, Staii C, Johnson A T, Pinto N J et al 2003 Appl. Phys. Lett. 833800

[10] Suman M, Tomara A K and Shyam K 2010 J. Alloys Compd. $\mathbf{5 0 8} 406$

[11] Bhajantri R F, Ravindrachary V, Harisha A, Ranganathaiah C and Kumaraswamy G N 2007 Appl. Phys. A 87797

[12] Khanna P K, Singh N, Charan S, Subbarao V, Gokhale R and Mulik U P 2005 Mater. Chem. Phys. 93117

[13] Seira M, Takashi N and Takuya G 2012 Polym. J. 441056

[14] Nejati K, Rezvani Z and Pakizevand R 2011 Int. Nano Lett. 175

[15] Zidan H M 2003 J. Appl. Polym. Sci. 881115

[16] Bhajantri R F, Ravindrachary V, Harisha A, Crasta V, Suresh Nayak P and Poojary B 2006 Polymer 473591

[17] Rithin Kumar N B, Vincent C, Praveen B M and Mohan K 2015 Nanotechnol. Rev. 4457

[18] Sherman R D, Middleman L M and Jacobs S M 1983 Polym. Eng. Sci. 2336
[19] Dutta A, Sinha T P, Jena P and Adak S 2008 J. Non-Cryst. Solids 3543952

[20] Kumar M, Tiwari T, Chauhan J K and Srivastava N 2014 Mater. Res. Express 1049601

[21] Pradhan D K, Choudhary R N P and Samantaray B K 2009 Mater. Chem. Phys. 115557

[22] Ruschau G R, Yoshikawa S and Newnham R E 1992 J. Appl. Phys. 72953

[23] Mahendia S, Tomar A K and Kumar S 2011 Mater. Sci. Eng. $B \mathbf{1 7 6} 530$

[24] Janzen J 1975 J. Appl. Phys. 46966

[25] Gonzalez-Campos J B, Prokhorov E, Sanchez I C, LunaBarcenas J G, Manzano-Ramirez A, Gonzalez-Hernandez J et al 2012 J. Nanomater. 1925750

[26] Paul E W, Riccio A J and Wrighton M S 1985 J. Phys. Chem. 89 1441

[27] Chanmal C V and Jog J P 2008 Express Polym. Lett. 2294

[28] Thomas P, Satapathy S, Dwarakanath K and Varma K B 2010 Express Polym. Lett. 4632

[29] Jitendra T, Khushbu Rahangdale K and Balasubramanian K 2016 RSC Adv. 669733

[30] Dutta P, Biswas S and De S K 2002 Mater. Res. Bull. 37193

[31] Bhadra S, Singha N K and Khastgir D 2009 Curr. Appl. Phys. 9 396

[32] Zheng P, Ling X K, Si-Dong L and Pavel S 2006 J. Nanosci. Nanotechnol. 63934

[33] Jun-Feng Z, Zhong-Zhen Y, Yu-Xun P, Xiao-Ping F and YuChun O 2002 J. Polym. Sci.: Part B: Polym. Phys. 40954

[34] Rachna M and Rao K J 2000 Ceram. Intl. 26371

[35] Cullis C F and Hirschler M M 1981 (Oxford: Clarendon Press)

[36] Anders H and Zimmerman H 1987 Polym. Degrad. Stab. 18 111 